ARTÍCULO

\title{
Análisis de Germinación de Semillas de Eucalyptus nitens Tratadas con Radiación Gamma: Indicios de Efecto Hormético.
}

\author{
Braulio Gutiérrez ${ }^{1 *}$; Laura Koch; Daniel Villegas²; Jorge Gonzalez¹ Doris Ly²; María Molina1; Patricio \\ Rojas $^{3} \&$ Ethel Velásquez². \\ ${ }^{1}$ Instituto Forestal, sede Biobío. Concepción. bgutierr@infor.cl, Ikoch@infor.cl, jgonzalez@infor.cl; mmolina@infor.cl \\ ${ }^{2}$ Comisión Chilena de Energía Nuclear. Centro de Estudios Nucleares La Reina. Santiago. daniel.villegas@cchen.cl; \\ doris.ly@cchen.cl; ethel.velasquez.cchen.cl \\ ${ }^{3}$ Instituto Forestal, sede Metropolitana. Santiago. parojas@infor.cl \\ * Autor de correspondencia
}

DOI: https://doi.org/10.52904/0718-4646.2021.554

Recibido: 01.11.2021; Aceptado 17.12.2021

\begin{abstract}
RESUMEN
Se analiza un ensayo de germinación en laboratorio para evaluar el efecto de cinco dosis de radiación gamma (10, 20, 30, 40 y 50 Gy) más un testigo sin irradiar, sobre los parámetros capacidad de germinación (CG), energía germinativa (EG) y periodo de energía (PE) de semillas de Eucalyptus nitens. Se concluye la existencia de un efecto significativo de radiohórmesis asociado al tratamiento de $10 \mathrm{~Gy}$, el cual aumenta la capacidad germinativa respecto al testigo sin irradiar. Ninguno de los tratamientos restantes resulta diferente al testigo en capacidad germinativa, energía germinativa ni periodo de energía.
\end{abstract}

Se comparan los resultados con los registrados en la bibliografía y se plantean recomendaciones para estudios posteriores en esta materia.

Palabras clave: Eucalyptus nitens, radiación gamma, hórmesis, germinación.

\section{SUMMARY}

A laboratory germination test is analyzed to evaluate the effect of five doses of gamma radiation (10, 20, 30, 40 and 50 Gy) plus a control without irradiation, on the parameters of germination capacity (GC), germination energy (GE) and energy period (EP) of Eucalyptus nitens seeds. The existence of a significant effect of radio-hormesis associated with the 10 Gy treatment is concluded. This treatment increases the germination capacity in relation to the not irradiated control. None of the remaining treatments is different from the control in germination capacity, germination energy or energy period.

The results are compared with those recorded in the bibliography. Recommendations are made for further studies in this area.

Keywords: Eucalyptus nitens, gamma radiation, hórmesis, germination

\section{INTRODUCCIÓN}

La inducción de mutaciones mediante radiación nuclear ha jugado un rol importante en el mejoramiento de diversas especies de cultivo agrícola (FAO/IAEA, 2020) ${ }^{1}$, sin embargo no ha sido utilizada en forma exitosa en especies forestales. El prolongado ciclo reproductivo de estas últimas especies (Gustafsson \& Mergen, 1964; Mukhtar et al., 2013; Riyal, 2011), así como la gran diversidad genética que ellas presentan, constituyen importantes desventajas y limitaciones para realizar mejoramiento genético vía mutagénesis inducida, técnica que es especialmente efectiva en cultivos con una base genética angosta,

\footnotetext{
${ }^{1}$ A noviembre del año 2021 la base de datos de IAEA registra 3.365 variedades de cultivo obtenidas por mutagénesis inducida por radiación nuclear. Aproximadamente la mitad de ellas corresponde a cereales; un $40 \%$ se distribuye entre flores, legumbres, plantas alimenticias y de aceite comestible; el porcentaje restante son diversas y variadas categorías, ninguna de las cuales corresponde a árboles forestales. (https://mvd.iaea.org).
} 
donde la diversidad alélica natural es limitada (Kumar, 2012; Jankowicz-Cieslak \& Till, 2015), situación precisamente opuesta a la presente en especies forestales.

Otro uso de la radiación ionizante, pero que no busca inducir cambios permanentes en el material genético, es la radio-hórmesis u hórmesis por radiación, consistente en la aplicación de pequeñas dosis de radiación gamma ${ }^{2}$ para generar distintos efectos biopositivos o respuestas de activación metabólica en los organismos irradiados (Luckey, 1980 cit. por Luckey y Lawrence, 2006).

Los primeros estudios en radio-hórmesis datan de las décadas de 1950-60 y señalan diversos efectos estimulantes de bajas dosis de radiación ionizante sobre cultivos vegetales de importancia agrícola (cereales, papas y cultivos forrajeros), en los que se observó estimulación de las tasas de germinación, mejoras en crecimiento radical y mayor velocidad de crecimiento inicial (FAO/IAEA, 1966). Con posterioridad y tras múltiples evaluaciones, estos efectos han sido confirmados en distintos parámetros asociados a cambios fisiológicos como la germinación de semillas, la estimulación/inhibición del crecimiento vegetal y otros (Piri et al., 2011; Calabrese, 2014). Sin embargo, el efecto de bajas dosis de radiación gamma sobre árboles forestales ha sido relativamente poco estudiado en los últimos 50 años.

Iglesias et al. (2012) abordan la importancia y el potencial del uso de bajas dosis de radiación gamma en estas especies, indicando que existen pocas referencias al uso de radio-hórmesis en árboles de importancia forestal. Aun así, existen antecedentes que indicarían diversas respuestas ante la estimulación con bajas dosis de radiación en especies forestales, entre ellas, Ferreira et al. (1980) describieron un efecto promotor de la germinación en semillas de Araucaria angustifolia tratadas con radiación gamma; González et al. (1999) indican estimulación en el crecimiento de raíz principal, número de hojas primaria y crecimiento del hipocotilo en plántulas de Pinus monctezumae; Iglesias et al. (2010) establecen que la aplicación de bajas dosis de radiación gamma en semillas de Abies religiosa produce un efecto positivo sobre altura de las plantas.

Con la finalidad de contribuir al conocimiento y dilucidar el grado de aplicación práctica que los efectos de la radio-hórmesis pudiesen tener en especies de interés forestal, en el presente artículo se presenta un trabajo preliminar con los primeros resultados obtenidos al analizar el efecto de bajas dosis de radiación gamma sobre los parámetros de germinación de semillas de Eucalyptus nitens (Deane \& Maiden) Maiden.

\section{MATERIAL Y MÉTODO}

El estudio de dosimetría para determinar las dosis de radiación a considerar en el ensayo de germinación, así como la irradiación de las semillas consideradas en el mismo, fueron efectuadas en el laboratorio de irradiaciones de la Comisión Chilena de Energía Nuclear, en Santiago, por profesionales pertenecientes a esa dependencia. Posteriormente, la instalación y evaluación propiamente tal del ensayo de germinación con la semilla irradiada que se evalúa en este artículo, se realizó en laboratorios del Instituto Forestal en Concepción.

\section{Determinación de las dosis de irradiación a ensayar}

Los tratamientos de irradiación fueron realizados en Irradiador Gammacell 220R, el cual cuenta con una fuente de Co60 con una actividad de 800 Curie (Ci) y un volumen de irradiación cilíndrico de 2 litros. Previo a la realización de los tratamientos, se caracterizó el volumen de irradiación mediante dosimetría Fricke formulada de acuerdo a Norma ASTM E-1026 e instructivos internos de CCHEN, estableciéndose una tasa de dosis de $8.98 \mathrm{~Gy} / \mathrm{min}$. Una vez establecida esta tasa de dosis y en función de la vida media o decaimiento del Co60 fue posible establecer con precisión para cualquier fecha, el tiempo de exposición de las semillas en el irradiador, para obtener una determinada dosis de radiación absorbida que se desee ensayar. A partir de estos antecedentes se realizó una curva dosimétrica con dosis crecientes $(0$ a 200 Gy) para determinar dosis letal DL50, nivel de irradiación por sobre la cual se genera una mortalidad del $50 \%$ de las semillas irradiadas, verificado como la pérdida de germinación respecto a semillas sin irradiar. La información obtenida permitió establecer que la DL50 para semillas de E. nitens se alcanza al irradiar

\footnotetext{
${ }^{2}$ Radiación electromagnética ionizante, de altísima frecuencia, consistente en fotones emitidos por elementos radiactivos
} 
con dosis de $160 \mathrm{~Gy}^{3}$. A partir de este dato y considerando que el 30\% de la DL50 constituye un límite práctico habitual para probar efectos horméticos, se definió como dosis a ensayar valores que cubriesen el rango entre 0 y 50 Gy.

El estudio de dosimetría y el desarrollo del protocolo de irradiación constituyen por si solos actividades de investigación que ameritan ser detallados y publicados como documentos independientes. Ellos involucran detalles metodológicos de alta complejidad que escapan al alcance de este artículo.

Para efectos de este estudio, la irradiación propiamente tal de las semillas que se utilizaron en el ensayo de germinación, se efectuó en ampollas de vidrio de $5 \mathrm{ml}$ (proveedor FAVIMA: www.favima.cl). En cada ampolla se depositó $0,1 \mathrm{~g}$ de semillas, aproximadamente 125 unidades, y posteriormente fueron irradiadas por el tiempo necesario para obtener las cinco dosis a evaluar (10, 20, 30, 40 y 50 Gy). Para disponer las ampollas en el irradiador se utilizó una plataforma de plumavit, de modo que ellas quedasen en el punto central de plano dosimétrico medio $(10 \mathrm{~cm})$ del irradiador.

\section{Montaje Ensayo de Germinación}

El ensayo de germinación se estableció en laboratorio, siguiendo las directivas de ISTA ${ }^{4}$ (2016). En él se comparó semilla de $E$. nitens sometida a cinco dosis de radiación ionizante, más un control sin irradiar (Cuadro 1). Cada tratamiento fue representado con tres repeticiones de 50 semillas cada una, las que fueron dispuestas en placas Petri con papel filtro humedecido previamente esterilizado. Cada placa se identificó con el número del tratamiento y la repetición (Figura 1); posteriormente fueron incubadas en cámara germinadora, en oscuridad, a temperatura de $22^{\circ} \mathrm{C}$ durante 20 días. Previo al montaje del ensayo, las semillas se dejaron en remojo por un día en agua destilada a temperatura ambiente.

Cuadro 1. Tratamientos de irradiación evaluados.

\begin{tabular}{cc}
\hline Tratamientos & Dosis de radiación \\
\hline T0 & Testigo sin irradiar \\
\hline T1 & $20 \mathrm{~Gy}$ \\
\hline T2 & $30 \mathrm{~Gy}$ \\
\hline T3 & $40 \mathrm{~Gy}$ \\
\hline T4 & $50 \mathrm{~Gy}$ \\
\hline T5
\end{tabular}

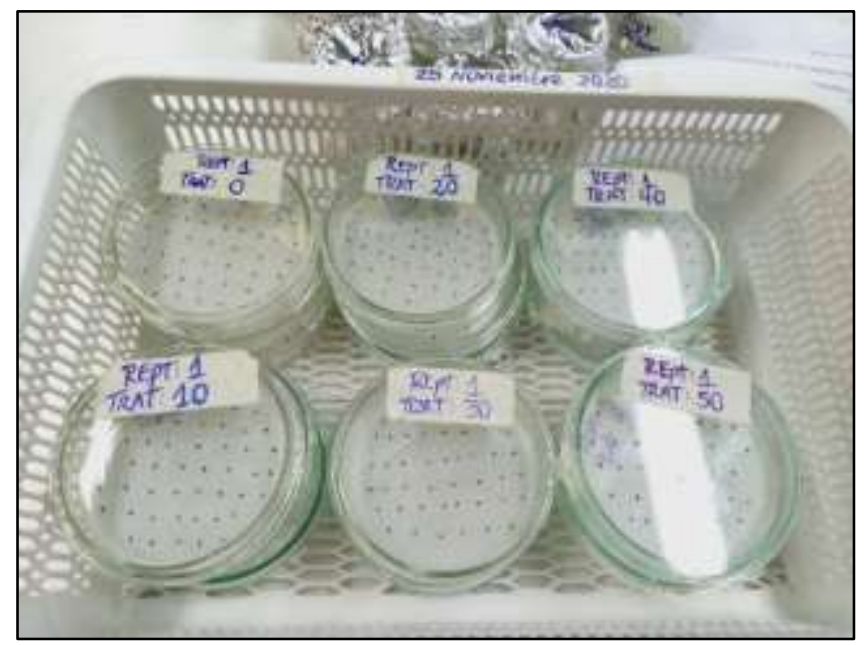

(Fotografía: L. Koch)

Figura $\mathbf{N}^{\circ}$. Ensayo de germinación de semillas irradiadas de $E$. nitens dispuesto en placas Petri

\footnotetext{
${ }^{3}$ Gray (Gy) es la unidad usada para representar la cantidad de energía radiante absorbida en cierta cantidad de tejido. Un Gray equivale a un Joule de energía absorbida por kilogramo de materia $(\mathrm{J} / \mathrm{Kg})$.

${ }^{4}$ Asociación Internacional para el Análisis de Semillas
} 


\section{Evaluación del ensayo de germinación}

El ensayo fue monitoreado hasta que se detuvo la germinación (20 días), registrándose diariamente, a la misma hora, el número de semillas germinadas en cada repetición de cada tratamiento, y retirándolas de las placas. Como criterio de germinación se asumió la presencia de radícula extendida en una longitud mayor a $2 \mathrm{~mm}$ (figura $\mathrm{N}^{\circ} 2$ ).

Con los datos de germinación diaria de las tres repeticiones de cada tratamiento, se construyó la curva de germinación acumulada diaria y a partir de ella se obtuvo los valores de los parámetros: Capacidad Germinativa (CG\%); Energía Germinativa (EG\%) y Periodo de Energía (PE) de acuerdo a la metodología tradicional del valor máximo de Czabator (1962). Al respecto conviene recordar, que la energía germinativa (EG) corresponde al porcentaje de germinación acumulado al momento en que la tasa de germinación alcanza su valor máximo. La cantidad de días requeridos para alcanzar este máximo es el parámetro denominado periodo de energía (PE); en tanto que la germinación final obtenida al final del ensayo es la capacidad germinativa (CG).

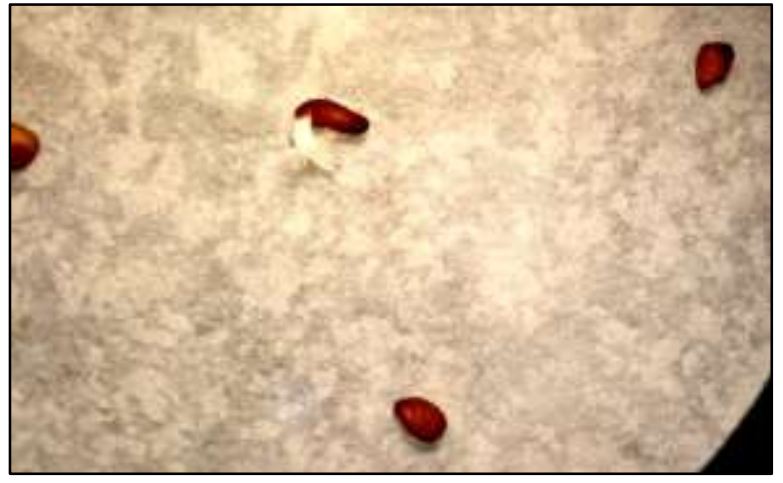

(Fotografía: L. Koch)

Figura 2. Semilla germinada de E. nitens

Para los tres parámetros mencionados se realizó análisis de varianza y pruebas de comparación múltiple de medias (Tuckey, alfa=0,05) para determinar la existencia de diferencias estadísticamente significativas entre tratamientos, utilizando el software Infostat 2015. Para efectos del análisis e interpretación estadístico, las variables expresadas en porcentaje (CG y EG) fueron previamente transformados a unidades de Bliss usando la transformación angular o del arcoseno, que resulta especialmente apropiada para análisis de varianza de variables en porcentajes o proporciones (Sokal y Rohlf, 1980; Mead et al., 2002). No obstante, para la presentación de resultados se conservan los valores en porcentaje.

\section{RESULTADOS}

Las curvas de germinación acumulada de los tratamientos ensayados (Figura 3) evidencian que todos ellos alcanzan valores similares, y que el testigo muestra un desempeño intermedio entre el de los tratamientos con bajas dosis de irradiación (10, y $20 \mathrm{~Gy}$ ) y el de los tratamientos con altas dosis de irradiación (40 y $50 \mathrm{~Gy}$ ). En general y como resulta habitual en semillas de E. nitens, esta presentó una alta germinación, tanto en el testigo como en todos los tratamientos de irradiación evaluados. 


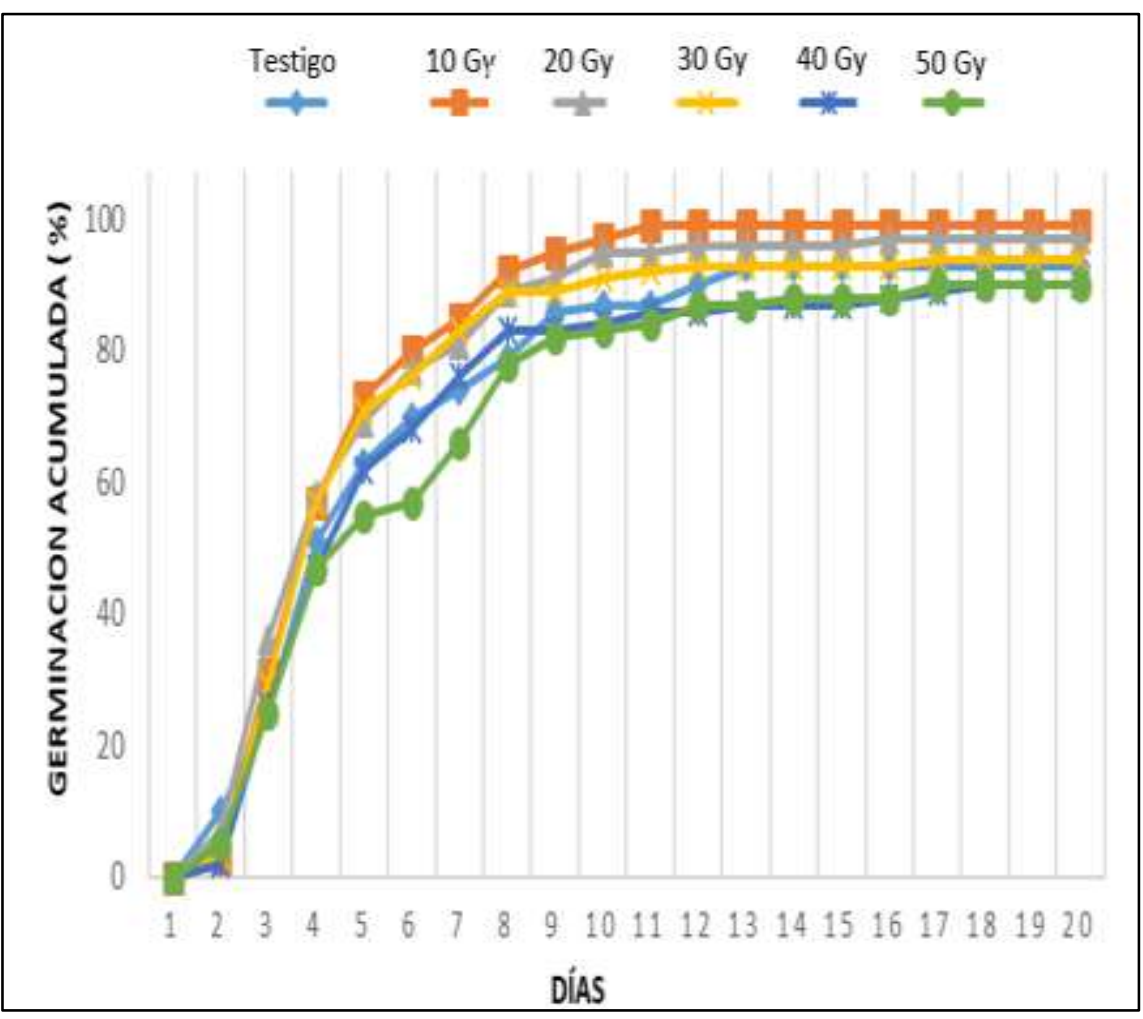

Figura $\mathbf{N}^{\circ}$ 3. Germinación acumulada de semillas de E. nitens tratadas con distintas dosis de radiación gamma

La capacidad germinativa total, que fluctúa entre 90 y $99 \%$, presenta diferencias significativas entre tratamientos $(p$-value $=0,0035)$, observándose una relación inversa entre dosis y capacidad de germinación (Figura 4-A). En este caso el testigo forma un grupo homogéneo con los tratamientos de 20, 30, 40 y 50 Gy, en tanto los tratamientos de 10 y 20 Gy constituyen un grupo distinto y de mayor germinación. Así, el tratamiento de menor dosis (10 Gy) resulta estadísticamente superior al testigo y a los tratamientos restantes, excepto al de 20 Gy que es la segunda menor dosis considerada en el ensayo. La máxima tasa de germinación o energía germinativa fluctúa entre 50 y $69 \%$ sin evidenciar diferencias estadísticamente significativas entre tratamientos y testigo (Figura 4-B), lo mismo ocurre con el periodo de energía, o tiempo requerido para lograr tal germinación, el que fluctúa entre 4,3 y 6 días (Figura 4-C) y tampoco exhibe diferenciación estadística significativa. 


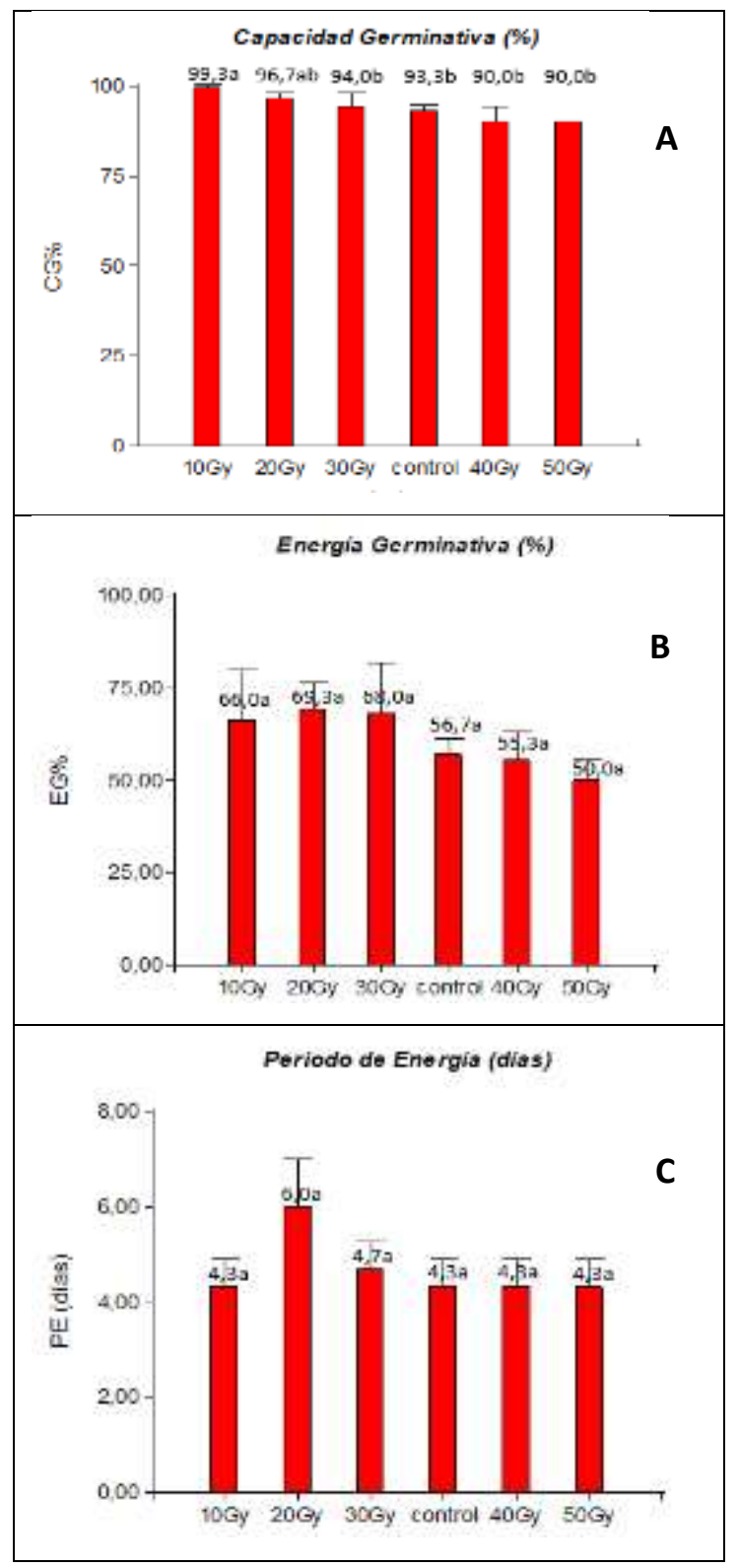

Figura 4. Valores medios por tratamiento, desviaciones estándar y diferenciación estadística para parámetros de germinación de semillas de $E$. nitens tratadas con distintas dosis de radiación gamma. Medias con una letra común no son significativamente diferentes (Tuckey, alfa $=0,05$ )

\section{DISCUSIÓN}

Los resultados obtenidos en el ensayo de germinación muestran que dosis de irradiación de 0 a 50 Gy no motivan efectos adversos sobre los parámetros de germinación de E. nitens; ninguna de ellas manifestó algún parámetro germinativo significativamente inferior al testigo sin irradiar. Por el contrario, se observa una diferenciación positiva de la dosis más baja (10 Gy), la que se hace evidente para la capacidad de germinación, pero que no exhibe diferencias respecto al testigo ni tratamientos restantes en términos de energía germinativa ni periodo de energía. 
Dicho resultado por sí solo es interesante ya que viene a reforzar la idea de que las plantas no responden a la radiación ionizante mediante un modelo linear (en que toda dosis es perjudicial) si no que presentan una respuesta que podría ser bifásica (bajas dosis podrían generar efectos positivos mientras que altas dosis ser dañinas) o no linear (presentar una tolerancia a bajas dosis de radiación) (Agathokleous et al., 2019).

La dosis más baja de entre las ensayadas (10 Gy) fue la única que mejoró en forma estadísticamente significativa la germinación de E. nitens, lo que, sumado a la relación inversa entre dosis y capacidad de germinación, sugiere que valores menores a 10 Gy serían aún más efectivas. En rigor, atendiendo a la tendencia general de las curvas de efecto hormético, a los valores de germinación obtenidos en el ensayo y al hecho de no haber probado dosis intermedias entre 0 y 10 Gy ni entre 10 y 20 Gy, se pueden presentar dos situaciones dependiendo de si la dosis óptima (DO) que maximiza el efecto positivo en la germinación se encuentra bajo o sobre los 10 Gy (Figura 5), la que sugiere que en general esta se encontraría bajo los 20 Gy. No obstante, debido a la gran capacidad de germinación de la especie y el alto nivel de germinación alcanzado con 10 Gy (99\%), no se justifica el esfuerzo de probar dosis intermedias para encontrar el valor óptimo de irradiación que maximice la germinación, pues en la práctica ya no queda margen para mejorar esta característica.

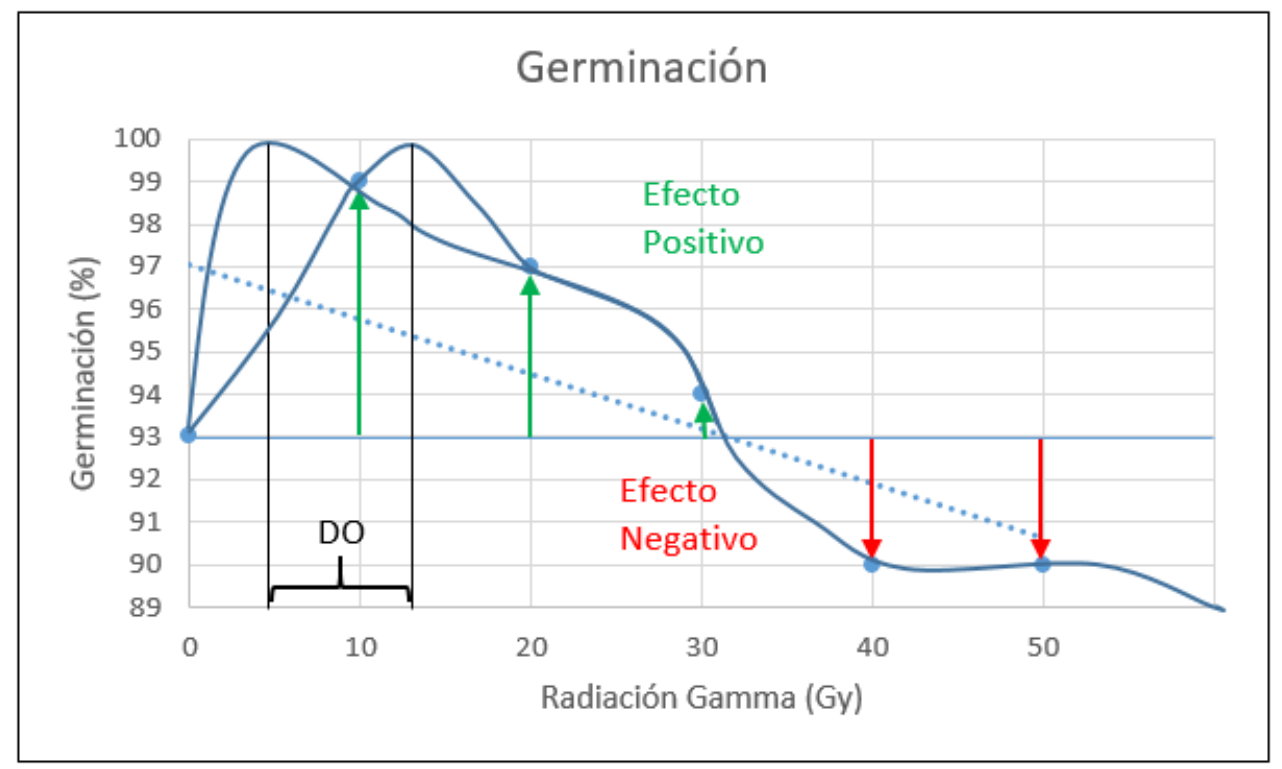

Figura 5. Posibles ubicaciones de la dosis óptima (DO) de irradiación para maximizar la germinación de semillas de $E$. nitens, de acuerdo a los resultados del ensayo evaluado

Dosis de radiación gamma inferiores a 10 Gy han producido efectos positivos y significativos sobre la germinación de semillas y crecimiento de plántulas de Pinus nigra, los que fueron obtenidos con dosis de irradiación de 0,88 a 8,88 Gy (Dudic, 1961 cit. por Ferreira et al., 1980). Sin embargo, los resultados no son consistentes entre especies, por cuanto en Pinus elliottii y Pinus taeda Da Vis (1962, cit. por Ferreira et al., 1980) no detecta diferencias significativas de germinación respecto al testigo, en semillas tratadas con un amplio espectro de dosis de radiación gamma comprendido entre 2,8 y 57,3 Gy.

Ensayos análogos realizados con semillas irradiadas de otras especies forestales, muestran resultados diversos, los que por tratarse de especies diferentes no son directamente comparables con los aquí obtenidos, pero que igualmente constituyen referencias a considerar. Iglesias et al. (2010) tras evaluar el efecto de la aplicación de radiación gamma en dosis de 2, 5, 10, 15 y 20 Gy sobre la germinación de semillas de Abies religiosa concluyen que las dosis bajas no se diferencian del testigo sin irradiar, en tanto que las dosis mayores presentan un efecto detrimental sobre la germinación y otras características de las plántulas. Tales resultados resultan idénticos a los informados por Dada et al. (2018) respecto al efecto sobre el vigor de plántulas de café tratadas con radiación gama en dosis de entre 0 y 25 Gy. 
Dosis inferiores a las evaluadas en este artículo fueron utilizadas por Ferreira et al. (1980) en Araucaria angustifolia, quienes observaron que dosis de hasta cerca de 5 Gy no tienen efecto significativo sobre la germinación de semillas, pero que las dosis menores (0,88 a 2,7 Gy) mejoraron el crecimiento de las plántulas después de 80 días de sembradas, respecto a las del testigo sin irradiar. En la misma especie, un estudio posterior de Modolo et al. (2011), donde se utilizan dosis de hasta 17,5 Gy, concluyen que solo las dosis más bajas (0,25 y 0,5 Gy) obtienen mejor germinación que el testigo, aun cuando esa diferencia no es estadísticamente significativa; y que a partir de 12,5 Gy el efecto resulta letal para las semillas.

En general, existen pocas referencias respecto al uso de radiación gamma en especies forestales y no es una técnica que se aplique en forma corriente. Iglesias et al. (2012) mencionan algunos antecedentes genéricos en especies forestales, entre ellos señalan que: dosis entre 0 y 900 Gy estimularían crecimiento de plántulas de Salix nigra; dosis de hasta 450 Gy estimularían germinación y tasa de crecimiento de Tectona grandis; radiación ionizante de baja intensidad reduciría el tamaño de los conos, germinación y altura de las plántulas de Pinus rigida; semillas irradiadas de Quercus alba tiene menor germinación y producen plantas con menor supervivencia y crecimiento en altura, y que además exhiben aberraciones visuales en la morfología de las hojas.

En el caso de Eucalyptus nitens, datos no publicados aportados por Gonzalez et al. (2021) indican que plantas de vivero obtenidas a partir de semillas irradiadas con 0, 10, 20 y 30 Gy presentaron diferencias estadísticamente significativas de altura y diámetro de cuello entre tratamientos, pero que ninguno de ellos fue mejor que el testigo sin irradiar; el mismo análisis señala que en términos de la proporción de la eficiencia fotosintética máxima, no existen diferencias con significancia estadística entre los distintos tratamientos y el testigo (Figura 6). Lo anterior estaría indicando que el positivo efecto observado en la dosis de 10 Gy sobre la germinación de E. nitens, no tendría incidencia en el desarrollo posterior de las plantas, confirmando la impermanencia del efecto hormético en el tiempo.

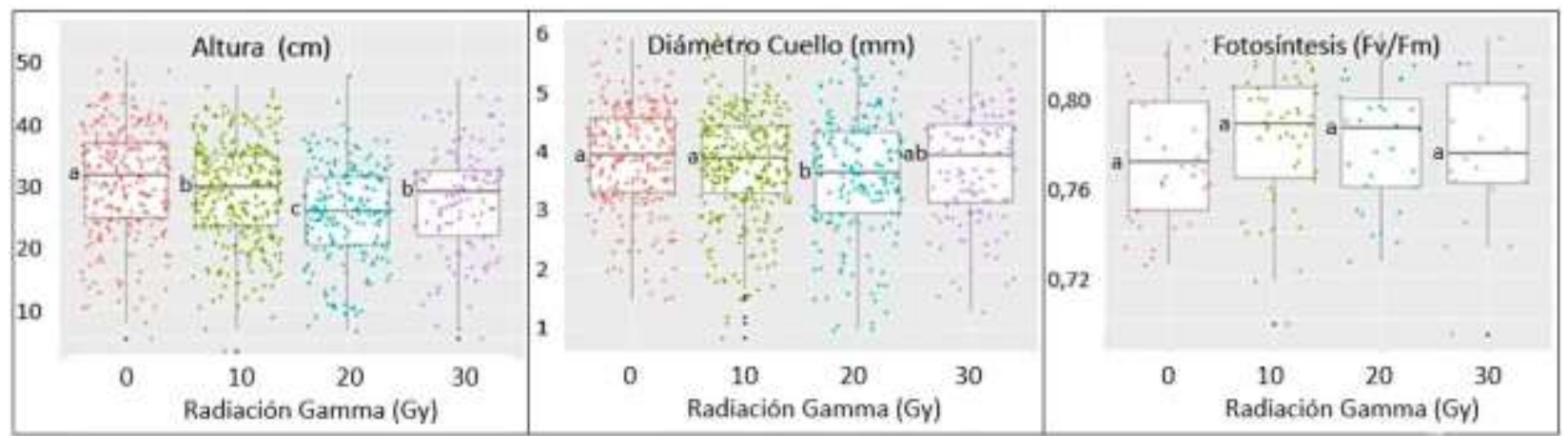

(Fuente: González et al., 2021)

Figura 6. Efecto sobre la altura, diámetro de cuello y fotosíntesis de plantas de Eucalyptus nitens de una temporada de viverización, obtenidas a partir de semillas irradiadas con 0, 10, 20 y 30 Gy de radiación Gamma.

\section{CONCLUSIONES}

Se concluye que dosis de 10 Gy de radiación gamma tiene un efecto positivo para mejorar la germinación de semillas de E. nitens; y que dosis de 20 a 50 Gy no exhiben diferencias respecto al testigo sin irradiar.

Puesto que E. nitens germina abundantemente (más del $90 \%$ en semillas testigo), es cuestionable la aplicación práctica y el valor operativo de este resultado. Aun así, el constatar que la radiación gamma efectivamente puede mejorar la germinación resulta un antecedente de interés para repetir los ensayos, pero con especies que presenten dificultades de germinación, de modo de poder validar en qué medida la aplicación de radiación pueda ser, en la práctica, un eficiente tratamiento pregerminativo.

Los resultados preliminares orientan respecto a la magnitud de la radiación gamma a utilizar y confirman que dosis relativamente bajas serían las más efectivas para mejorar la germinación, pero atendiendo a las diferencias entre especies y a la diversidad de resultados existentes en la bibliografía, las dosis a probar deben ser definidas para cada especie en particular. 
El presente estudio analizó la respuesta a los tratamientos de radiación sobre la germinación de semillas, tópico que junto a los efectos sobre el crecimiento de plántulas constituyen la mayoría de los estudios en esta materia. Futuras investigaciones deberán consolidar la información en estos procesos básicos, pero también ampliarse a los cambios a nivel fisiológico; bioquímico y/o molecular que puedan manipularse mediante radio-hórmesis.

\section{AGRADECIMIENTOS}

Al proyecto "Evaluación de la radio-hórmesis como metodología para el mejoramiento de parámetros de crecimiento en las especies Eucalyptus nitens y Peumus boldus" del Programa de Atracción e Inserción de Capital Humano Avanzado, de CONICYT; al proyecto CHI 5052 "Application of nuclear, molecular and biotechnological techniques to improve the adaptation and growth of native and exotic forest species facing climate change", financiado por la Agencia Internacional de la Energía Atómica (IAEA); y a la empresa Forestal Mininco S.A., en la persona de la Sra. Verónica Emhart S., por el suministro de semillas para estas investigaciones.

\section{REFERENCIAS}

Agathokleous, E., Kitao, M. \& Calabrese, E.J. (2019). Hormesis: a compelling platform for sophisticated plant science. Trends in Plant Science, 24(4): 318-327. https://doi.org/10.1016/j.tplants.2019.01.004

Calabrese, E. (2014). Hormesis: a fundamental concept in biology. Microbial Cell, 1(5): 145-149. https://doi.org/10.15698/mic2014.05.145

Czabator, F.J. (1962). Germination value: an index combining speed and completeness of pine seed germination. Forest Science, 8(4): 386-396.

Dada K.E., Anagbogu, C.F., Forster, B.P., Muyiwa, A.A., Adenuga, O.O., Olaniyi, O.O. \& Bado, S. (2018). Biological effect of gamma irradiation on vegetative propagation of Coffea arabica L. African Journal of Plant Science, 12(6): 122-128. https://doi.org/10.5897/AJPS2016.1504

FAO/IAEA. (1966). Effects of low doses of radiation on crop plants. Technical Report Series N 64. Vienna. 58 p.

FAO/IAEA. (2020). Mutant variety database. International Atomic Energy Agency. En: https://mvd.iaea.org. Consulta: 3 junio, 2020.

Ferreira, C., Franco do Nascimiento, V., Ferreira, M. \& Vencovsky, R. (1980). Efeito de baixas doses de radiação gama na conservação do poder germinativo de sementes de Araucaria angustifolia (Bert). IPEF № 21. Pp: 6782.

González, J., Jimenez, M. \& García, M. (1999). Mejoramiento genético del pino real (Pinus montezumae Lamb). Parte I. En: https://inis.iaea.org/collection/NCLCollectionStore/_Public/32/022/32022749.pdf. Consulta: enero, 2021.

Gonzalez, J., Koch, L. \& Vera, T. (2021). Ensayo hórmesis de Eucalyptus nitens. Presentación interna (no publicada). Instituto Forestal, Línea Mejoramiento Genético y Conservación. Concepción. 29 p.

Gustafsson, A. \& Mergen, F. (1964). Some principles of tree cytology and genetics. Unasylva, 18(2-3): En: http://www.fao.org/docrep/03650e/03650e02.htm\#1.\%20some\%20principles\%20of\%20tree\%20cytology\%20an d\%20genetics.

Iglesias, L., Sánchez, R., Tivo, Y., Luna, M., Flores, N., Noa, J., Ruiz, C. \& Moreno, J. (2010). Efecto de radiaciones gamma en Abies religiosa (Kunth) Schltd. et Cham. Rev. Chapingo Ser. Cienc. For. Ambient. Vol.16 N¹ Chapingo ene. /jun.

Iglesias, L., Aguilar, O. \& Bello, J. (2012). Current importance and potential use of low doses of gamma radiation in forest species. En: INTECH (Editor). 2012. Gamma radiation. Capítulo 13.

ISTA. (2016). Reglas internacionales para el análisis de las semillas. Introducción a las reglas ISTA Capítulos 1-7, 9. International Seed Testing Asociation (ISTA), Zurichstr. Basserrsdorf, Suiza. Online ISSN 2310-3655.

Jankowicz-Cieslak, J. \& Till, B. (2015). Forward and Reverse Genetics in Crop Breeding. En: Al-Khayri, J.M. et al. (Eds). Advances in Plant Breeding Strategies: Breeding, Biotechnology and Molecular Tools. Springer International Publishing. Switzerland. https://doi.org/10.1007/978-3-319-22521-0_8

Kumar, J. (2012). Studies on induction of mutations in Swertia chirayita (Roxb. Ex. Flem.) H. Karst. Thesis Submitted in partial fulfillment of the requirements for the degree of Master of Science in Forestry (forest products). 
College of Forestry Dr. Yashwant Singh Parmar University of Horticulture and Forestry, Nauni, Solan, INDIA. $106 \mathrm{p}$.

Luckey, T.D. \& Lawrence, K.S. (2006). Radiation Hormesis: the good, the bad, and the ugly. International Hormesis Society. Dose-Response, 4(3): 169-190. https://doi.org/10.2203/dose-response.06-102.Luckey

Mead, R., Curnow, R.N. \& Hasted, A.M. (2002). Statistical methods in agriculture and experimental biology. $3^{\text {rd }}$ ed. Ed. Chapman \& Hall/CRC. Boca Raton, FL. 472 p.

Modolo, D., Silva, L., Harder, M., Arthur, P. \& Arthur, V. (2011). Effects of low doses of gamma irradiation on pine nuts (Araucaria angustifolia). In: International Nuclear Atlantic Conference, INAC. Bello Horizonte, MG, Brazil. 2428 october, 2011.

Mukhtar, A., Ghanim, A. \& Forster, B. (2013). Techniques for acceleration of mutation breeding in crop plants. Tagung der Vereinigung der Pflanzenzüchter und Saatgutkaufleute Österreichs 2013, 7. ISBN: 978-3-90284900-7.

Piri, I., Babayan, M., Tavassoli, A. \& Javaheri, M. (2011). The use of gamma irradiation in agriculture. African Journal of Microbiology Research, 5(32): 5806-5811. https://doi.org/10.5897/AJMR11.949

Riyal, D. (2011). Development of mutation-based breeding technology in forest tree species. Master of Science Thesis. Simon Fraser University, Department of Biological Science. Burnaby, BC, Canada. 245 p.

Sokal, R. \& Rohlf, FJ. (1980). Introducción a la bioestadística. Edición española. Editorial Reverte S.A. Barcelona, España. 